\title{
Proton emission - new results and future prospects
}

\author{
R. D. Page ${ }^{1, a}$ \\ ${ }^{1}$ Department of Physics, Oliver Lodge Laboratory, University of Liverpool, Liverpool L69 7ZE, United Kingdom
}

\begin{abstract}
Proton emission is the radioactive decay mode that is expected to determine the limit of observable proton-rich nuclei for most elements. Considerable progress has been made in the study of proton-emitting nuclei since the first observation of direct proton emission nearly 50 years ago. This has led to improvements in our understanding of this decay process and provided invaluable nuclear structure data far from the valley of beta stability. The rapid fall in half-lives with increasing neutron deficiency when proton emission dominates makes it likely that for some elements, the lightest isotopes whose ground states can be observed in conventional experiments have already been reached. The enhanced stability against proton emission of the recently discovered high-lying isomer in ${ }^{158} \mathrm{Ta}$ raises the possibility that proton emission from multiparticle isomers could be observed in nuclei beyond the expected boundaries of the nuclear landscape.
\end{abstract}

\section{Introduction}

Isomers are a widespread feature across the chart of nuclides and their study can provide an important testing ground for a range of nuclear theories [1]. The existence of isomeric states is also an important consideration in the study of nuclei beyond the proton drip line and is inextricably linked to the question of the limits of observable nuclei. The first experimentally observed case of direct proton emission was from a high-spin isomer in ${ }^{53} \mathrm{Co}[2,3]$. In this unique case, the proton emitter's ground state is bound to proton emission, but the excitation energy of the protonunbound isomer is sufficient to overcome the retarding effect of the centrifugal barrier and results in a proton-decay branching ratio of $\sim 1.5 \%$. Subsequent studies of proton emission have primarily focused on low-lying states in nuclei beyond the proton drip line. As these studies reach the limits of nuclei that are accessible using recoil separators, attention may again switch to proton emission from highlying isomeric states, in nuclei far beyond the proton drip line.

\section{Proton emission from low-lying states}

The discovery of the first cases of proton emission from nuclear ground states [4, 5] followed a decade after the ${ }^{53}$ Co results and many further proton emitters have been discovered since [6]. Approximately half of the presently known cases of direct one-proton emission have been found in the region beyond the proton drip line that is bounded by the $N=82$ and $Z=82$ shell closures. In total, 21 cases of direct proton emission in this region have been reported to date. One reason for this rich sample is that either the proton-decaying states can be populated

\footnotetext{
a e-mail: rdp@ns.ph.liv.ac.uk
}

through alpha decays or the daughter nuclides are alpha emitters. This makes searches for proton-decay peaks with low numbers of counts very sensitive, because the alpha decays have characteristic energies that can be cleanly selected for correlation analyses and they tend to have fairly short half-lives, which result in relatively low levels of background from false correlations.

The structure of nuclei in this region is governed at low excitation energies by valence neutrons in the $v f_{7 / 2}$ and $v h_{9 / 2}$ orbitals and protons in the $\pi s_{1 / 2}, \pi d_{3 / 2}$ and $\pi h_{11 / 2}$ orbitals. In odd- $Z$ nuclides, two states are generally found at low excitation energies that can decay by proton, alpha or beta emission (see Fig. 1a). In odd- $A$ nuclei, the states usually have either an unpaired $s_{1 / 2}$ or $h_{11 / 2}$ proton, while in odd-odd nuclei either a $d_{3 / 2}$ or $h_{11 / 2}$ proton is coupled with an $f_{7 / 2}$ neutron to form the low-lying states. According to the Nordheim rules [7, 8], the lowest-energy state formed by coupling a $d_{3 / 2}$ proton with an $f_{7 / 2}$ neutron would have spin and parity $2^{-}$, while the lowest-energy state when coupling an $h_{11 / 2}$ proton with an $f_{7 / 2}$ neutron would be $9^{+}$. The energy differences between the states have been found to be $\leq 300 \mathrm{keV}$ (see Fig. 1b), so direct gamma decays from the isomeric state to the ground state in both odd- $A$ and odd-odd nuclides would be very slow owing to the large spin difference. Consequently charged particle emission generally dominates from the low-lying isomeric states and approximately half of the known cases of proton emission in this region are from these isomers, with the remainder being from ground states.

This pattern of two low-lying states is generally borne out, as can be seen in Fig. 1a, except for a few of the most exotic nuclei where only the $\pi h_{11 / 2}$ state has been observed. This is thought to be because the lowspin ground states are too short-lived to survive the flight time through a recoil separator $[12-15,17,18]$. The 


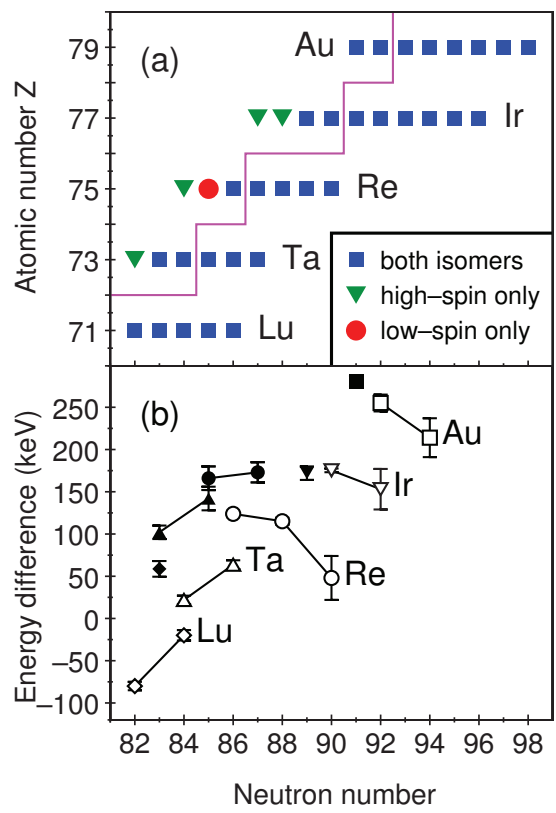

Figure 1. (a) Chart indicating the observed low-lying isomers in odd- $Z$ nuclei. The triangles indicate nuclei in which only the high-spin isomer has been observed, the squares indicate those in which both the high-spin and low-spin isomers have been observed and the circle indicates ${ }^{160} \mathrm{Re}$, which is the only nuclide in this region where only the low-spin isomer has been observed. Nuclides to the left of the line are known proton emitters. (b) Energy differences between the $\pi h_{11 / 2}$ isomers and the low-spin $\left(\pi s_{1 / 2}\right.$ or $\left.\pi d_{3 / 2}\right)$ isomers. The low-spin isomers represent the ground states of the nuclides shown, except for the Lu isotopes for which the $\pi h_{11 / 2}$ configuration forms the ground state. The open symbols denote odd- $A$ nuclei, while odd-odd nuclei are indicated by the filled symbols. Data are taken from Refs. [9-16].

only other exception is the case of ${ }^{160} \mathrm{Re}$, for which proton and alpha-particle emission have only been observed from the $d_{3 / 2}$ ground state [19-21]. In this nuclide a 2.8microsecond gamma-decaying isomer was identified [20] and subsequent in-beam gamma-ray spectroscopy measurements suggested that the isomer was based on a $\pi h_{11 / 2}$ orbital [22]. The discovery of the alpha-decay branch from the $\pi h_{11 / 2}$ state in ${ }^{164}$ Ir established the excitation energy of the corresponding state in ${ }^{160} \mathrm{Re}$, which was interpreted as being the isomeric state [15]. It seems that in ${ }^{160} \mathrm{Re}$ the multiplets of states formed by coupling the odd neutron and proton are so arranged as to allow a gamma-ray decay path that has a much shorter half-life than either proton or alpha emission from the $\pi h_{11 / 2}$ isomeric state [20].

Measurements of proton-decay energies can be used to deduce $Q_{\mathrm{p}}$ values that can be compared with predictions of atomic mass models. The systematics of $Q_{\mathrm{p}}$ values determined from proton-decay measurements on elements from $\mathrm{Tm}$ to $\mathrm{Tl}$ are shown in Fig. 2. For Ta, Re, Ir, Au and $\mathrm{Tl}$ proton emitters, the prevalence of extensive alpha-decay chains above $N=82$ allows $Q_{\mathrm{p}}$ values to be determined indirectly for states that do not themselves have a known proton-decay branch. This is in contrast to the protonemitting Tm and Lu nuclides whose $Q_{\mathrm{p}}$ values cannot be used in this way to deduce values for other nuclides. The

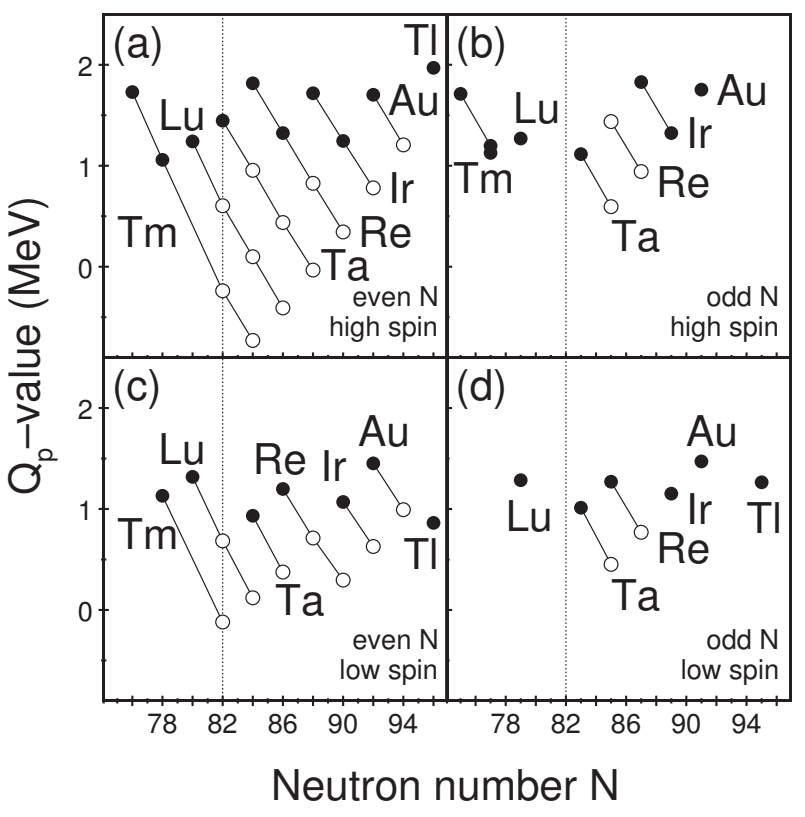

Figure 2. Experimental proton-decay Q-values of odd- $Z$ elements from $\mathrm{Tm}$ to $\mathrm{Tl}$ measured directly are shown by the filled circles. Values estimated by combining these values with $\alpha$ decay measurements are indicated by the empty circles. (a) is for high-spin $\left(\pi h_{11 / 2}\right)$ states in odd- $Z$, even- $N$ nuclei, while (b) shows the corresponding values for odd- $Z$, odd- $N$ nuclei. (c) and (d) show the values for low-spin $\left(\pi s_{1 / 2}\right.$ or $\left.\pi d_{3 / 2}\right)$ states in odd- $Z$, even- $N$ and odd- $Z$, odd- $N$ nuclei, respectively. Data are taken from Refs. [4, 5, 9-18, 21, 23-32].

smooth behaviour of the $Q_{\mathrm{p}}$ values as a function of neutron number allows local extrapolations of the trends to be made, which can be used to predict the decay properties of as yet unobserved states [33]. This makes it possible to identify good candidates for new proton emitter searches and to evaluate whether the limits of observable nuclei using conventional recoil separator techniques may have been reached.

\section{Proton emission from high-lying isomers}

In addition to the low-lying isomeric states discussed in the previous section, higher-lying multiparticle isomers are also known in this region. For example, seniority 3 $\left[\pi h_{11 / 2}^{3}\right] 27 / 2^{-}$isomers have been identified in the $N=82$ isotones ${ }^{149} \mathrm{Ho},{ }^{151} \mathrm{Tm}$ and ${ }^{153} \mathrm{Lu}[34-36]$. The possibility of proton emission from the corresponding isomer in ${ }^{155} \mathrm{Ta}$ to states in ${ }^{154} \mathrm{Hf}$ is discussed in Ref. [18]. The proton decay directly to the ground state of ${ }^{154} \mathrm{Hf}$ would be favoured by having the largest $Q_{\mathrm{p}}$ value, but be hindered by the large centrifugal barrier associated with the spin difference between the initial and final states. Decays to excited, higher-spin states in ${ }^{154} \mathrm{Hf}$ would be less hindered because the centrifugal barrier would be lower, but the excitation energy would reduce the effective $Q_{\mathrm{p}}$ value. In general, the balance between these competing effects will 
determine which proton-decay paths are most favoured in the decay of a given isomer.

Multiparticle isomers that decay by alpha emission have been established in $N=84$ isotones [16, 37, 38]. In even-even nuclei these isomers are interpreted as having a $\left[v f_{7 / 2} v h_{9 / 2}\right] 8^{+}$structure, while in the odd- $Z$ isotones the $\pi h_{11 / 2}$ proton couples with this configuration to give an isomeric $25 / 2^{-}$state. Both ${ }^{155} \mathrm{Lu}$ and ${ }^{157} \mathrm{Ta}$ are unbound to proton emission (see Fig. 2) and the probability of proton-decay branches to states in their respective daughter nuclides are analysed in Ref. [39]. In the case of ${ }^{155} \mathrm{Lu}$, proton emission from the isomer would be faster than from lower-lying states, which are only just unbound, whereas for ${ }^{157} \mathrm{Ta}$ all proton-decay paths from the isomer have longer partial half-lives than low-lying states.

The alpha and gamma decays of an even more complicated isomeric state in ${ }^{158}$ Ta have been reported recently $[39,40]$. A possible structure for this isomer is $\left[\pi h_{11 / 2}^{-3} v f_{7 / 2} v h_{9 / 2} v i_{13 / 2}\right] 19^{-}$, analogous to that proposed for an isomer in ${ }^{152} \mathrm{Ho}$ [41]. This isomer in ${ }^{158} \mathrm{Ta}$ lies at an excitation energy of $2668 \mathrm{keV}$ and although it is unbound to proton emission by $3261 \mathrm{keV}$, no proton-decay branch could be identified [39]. Analysis of the predicted lifetimes for proton-decay paths to states in the daughter nuclide ${ }^{157} \mathrm{Hf}$ revealed that none of them would be sufficiently short to compete with the other decay branches. Indeed, in contrast to the case of ${ }^{53} \mathrm{Co}$, the direct proton decay of the $19^{-}$isomer to the $7 / 2^{-}$ground state of ${ }^{157} \mathrm{Hf}$ would have a longer partial half-life than either of its lower-lying states.

\section{Conclusions}

Experimental studies of odd- $Z$ nuclei have identified more than 30 proton-emitting nuclei, with examples known for most elements from iodine $(Z=53)$ to bismuth $(Z=83)$. These studies have revealed how the half-lives of lowlying states generally decrease with increasing neutron deficiency, dropping more rapidly when proton or alpha emission dominates over beta decay. For some elements it appears likely that the lightest isotopes whose ground states can be observed in conventional recoil separator experiments have already been reached.

The enhanced stability against proton emission of the high-lying isomers in ${ }^{157,158} \mathrm{Ta}$ [39] raises the possibility that multiparticle isomers could provide observable states in nuclei whose low-lying states decay by proton emission too quickly to be studied with a recoil separator. Calculations suggest that multiparticle isomers in neutron-rich nuclei [42, 43] and superheavy nuclei [44] may also have enhanced stability. Decays of long-lived isomers beyond the expected boundaries of the nuclear landscape are an intriguing prospect for future experiments.

\section{Acknowledgements}

This work was supported by the United Kingdom Science and Technology Facilities Council.

\section{References}

[1] P. M. Walker and G. D. Dracoulis, Nature (London) 399, 35 (1999).

[2] K. P. Jackson et al., Phys. Lett. B 33, 281 (1970).

[3] J. Cerny et al., Phys. Lett. B 33, 284 (1970).

[4] S. Hofmann et al., Z. Phys. A 305, 111 (1982).

[5] O. Klepper et al., Z. Phys. A 305, 125 (1982).

[6] B. Blank and M. J. G. Borge, Prog. Part. Nucl. Phys. 60, 403 (2008).

[7] L. W. Nordheim, Phys. Rev. 78, 294 (1950).

[8] C. J. Gallgher Jr. and S. A. Moszkowski, Phys. Rev. 111, 1282 (1958).

[9] R. J. Irvine et al., Phys. Rev. C 55, 1621 (1997).

[10] H. Kettunen et al., Phys. Rev. C 69, 054323 (2004).

[11] G. L. Poli et al., Phys. Rev. C 59, 2979 (1999).

[12] H. Kettunen et al., Acta Phys. Pol. B 32, 989 (2001).

[13] H. Mahmud et al., Eur. Phys. J. A 15, 85 (2002).

[14] C. N. Davids et al., Phys. Rev. C 55, 2255 (1997).

[15] M. C. Drummond et al., Phys. Rev. C 89, 064309 (2014).

[16] R. D. Page et al., Phys. Rev. C 53, 660 (1996).

[17] D. T. Joss et al., Phys. Lett. B 641, 34 (2006).

[18] R. D. Page et al., Phys. Rev. C 75, 061302 (2007).

[19] R. D. Page et al., Phys. Rev. Lett. 68, 1287 (1992).

[20] I. G. Darby et al., Phys. Lett. B 695, 78 (2011).

[21] I. G. Darby et al., Phys. Rev. C 83, 064320 (2011).

[22] P. J. Sapple et al., Phys. Rev. C 84, 054303 (2011).

[23] R. Grzywacz et al., Eur. Phys. J. A 25, 145 (2005).

[24] K. Livingston et al., Phys. Lett. B 312, 46 (1992).

[25] M. Karny et al., Phys. Rev. Lett. 90, 012502 (2003).

[26] A. P. Robinson et al., Phys. Rev. C 68, 054301 (2003).

[27] J. C. Batchelder et al., Phys. Rev. C 57, 1042 (1998).

[28] C. R. Bingham et al., Phys. Rev. C 59, 2984 (1999).

[29] T. N. Ginter et al., Phys. Rev. C 61, 014308 (1999).

[30] T. N. Ginter et al., Phys. Rev. C 68, 034330 (2003).

[31] P. J. Sellin et al., Phys. Rev. C 47, 1933 (1993).

[32] P. O. Larsson et al., Z. Phys. A 314, 9 (1983).

[33] R. D. Page, Phys. Rev. C 83, 014305 (2011).

[34] H. Helppi et al., Phys. Lett. B 115, 11 (1982).

[35] J. H. McNeill et al., Phys. Rev. Lett. 63, 860 (1989).

[36] J. H. McNeill et al., Z. Phys. A 344, 3 (1993).

[37] S. Hofmann et al., Z. Phys. A 291, 53 (1979).

[38] S. Hofmann et al., Z. Phys. A 333, 107 (1989).

[39] R. J. Carroll et al., Phys. Rev. Lett. 112, 092501 (2014).

[40] R. J. Carroll et al., Phys. Rev. C 93, 034307 (2016).

[41] S. André et al., Nucl. Phys. A575, 155 (1994).

[42] L. K. Peker et al., Phys. Lett. B 36, 547 (1971).

[43] V. P. Bugrov et al., Sov. Jour. Nucl. Phys. 42, 34 (1985).

[44] F. R. Xu et al., Phys. Rev. Lett. 92, 252501 (2004). 